\title{
Characterization of polysaccharides extracted from spent coffee grounds by alkali pretreatment
}

\author{
Lina F. Ballesteros ${ }^{a}$, Miguel A. Cerqueira ${ }^{a}$, José A. Teixeira ${ }^{a}$, Solange I. Mussatto ${ }^{\text {b,* }}$ \\ a Centre of Biological Engineering, University of Minho, Campus Gualtar, 4710-057 Braga, Portugal \\ b Department of Biotechnology, Delft University of Technology, Julianalaan 67, 2628 BC Delft, The Netherlands
}

\section{A R T I C L E I N F O}

\section{Article history:}

Received 12 November 2014

Received in revised form 18 February 2015

Accepted 9 March 2015

Available online 1 April 2015

\section{Keywords:}

Antimicrobial activity

Antioxidant capacity

Polysaccharides

Spent coffee grounds

Structural properties

\begin{abstract}
A B S T R A C T
Spent coffee grounds (SCG), obtained during the processing of coffee powder with hot water to make soluble coffee, are the main coffee industry residues and retain approximately seventy percent of the polysaccharides present in the roasted coffee beans. The purpose of this study was to extract polysaccharides from SCG by using an alkali pretreatment with sodium hydroxide at $25^{\circ} \mathrm{C}$, and determine the chemical composition, as well as the antioxidant and antimicrobial properties of the extracted polysaccharides. Galactose $(60.27 \% \mathrm{~mol})$ was the dominant sugar in the recovered polysaccharides, followed by arabinose $(19.93 \% \mathrm{~mol})$, glucose $(15.37 \% \mathrm{~mol})$ and mannose $(4.43 \% \mathrm{~mol})$. SCG polysaccharides were thermostable, and presented a typical carbohydrate pattern. Additionally, they showed good antioxidant activity through different methods and presented high antimicrobial percent inhibition against Phoma violacea and Cladosporium cladosporioides (41.27\% and 54.60\%, respectively). These findings allow identifying possible applications for these polysaccharides in the food industry.
\end{abstract}

(c) 2015 Elsevier Ltd. All rights reserved.

\section{Introduction}

Polysaccharides are the main constituents of the coffee beans and play an important role in the final beverage. During the preparation of soluble coffee, the polysaccharides present in the roasted coffee are partially extracted with hot water and are responsible for the retention of coffee volatile substances, stabilization of foam, binding of aroma, formation of sedimentation, and increased viscosity of the extract (Arya \& Rao, 2007; Nunes, Domingues, \& Coimbra, 2005). Arabinogalactan, galactomannan and cellulose are the dominant polysaccharides in coffee beans (Arya \& Rao, 2007; Fischer, Reimann, Trovato, \& Redgwell, 2001). Arabinogalactans is the most significant group of polysaccharides extracted with hot water from green coffee (Arya \& Rao, 2007; Nunes et al., 2005). Nevertheless, after roasting process, galactomannans become the most relevant polysaccharides in roasted coffee infusions. Thus, galactomannans and arabinogalactans are the most important coffee constituents after hot water extraction.

Recently, some researchers have exposed the great potential of polysaccharides presented in coffee, showing that they can provide enormous functional properties. Most of these polysaccharides are

\footnotetext{
* Corresponding author. Tel.: +31 015278 8789; fax: +31 0152782355 .

E-mail addresses: S.I.Mussatto@tudelft.nl, solangemussatto@hotmail.com (S.I. Mussatto).
}

not degraded by human digestive enzymes; thus, they reach the colon and potentially serve as substrates for the colonic microbiota supporting the growth of bifidobacteria and other lactic acid bacteria that are considered beneficial for human health (Gniechwitz, Reichardt, Blaut, Steinhart, \& Bunzel, 2007). Polysaccharides from coffee decrease the cholesterol levels in blood, controlling the blood glucose and insulin response and act against infectious and tumor diseases (Gniechwitz et al., 2007; Simões et al., 2009). Additionally, polysaccharides from natural sources, especially galactomannans are excellent stiffeners and stabilizers of emulsions that can be used in food, pharmaceutical and cosmetic industries since they do not have toxic substances (Cerqueira et al., 2009; Vieira, Mendes, Gallão, \& de Brito, 2007). All these biological and physicochemical properties offered by coffee polysaccharides can be found in the main residue obtained during the processing of coffee powder with hot water to prepare instant coffee, known as the spent coffee grounds (SCG), which retain about $70 \%$ of total polysaccharides present in roasted coffee (Arya \& Rao, 2007).

Nowadays, the market of the soluble coffee around the world is increasing, which in turn generates around 6 million tons of SCG per year (Mussatto, Machado, Martins, \& Teixeira, 2011). Some studies have been proposed to exploit this residue, which is not only rich in polysaccharides but also in proteins, phenolic compounds among other components, being of great interest to chemical and food industries. SCG could be used, for example, to produce fuel for industrial boilers due to its high calorific power of approx. 
$5000 \mathrm{kcal} / \mathrm{kg}$ (Silva, Nebra, Machado Silva, \& Sanchez, 1998) and fuel ethanol (Mussatto, Machado, Carneiro, \& Teixeira, 2012), to produce mannitol (Arya \& Rao, 2007; Mussatto, Carneiro, Silva, Roberto, \& Teixeira, 2011), and as raw material to obtain antioxidant phenolic compounds (Machado, Rodriguez-Jasso, Teixeira, \& Mussatto, 2012; Mussatto, 2015; Mussatto, Ballesteros, Martins, \& Teixeira, 2011) or to produce a distilled beverage with aroma of coffee (Sampaio et al., 2013). Despite these possible applications, SCG are still underutilized as valuable material for industrial processes.

Some studies have reported the extraction of polysaccharides from coffee (green and roasted) and SCG by different techniques such as solid-liquid extraction with organic solvents (Fischer et al., 2001; Simões et al., 2009; Simões, Nunes, Domingues, \& Coimbra, 2010), dilute acid hydrolysis (Mussatto, Carneiro, et al., 2011) and microwave-assisted extraction (Passos \& Coimbra, 2013), but to the best of our knowledge, there is no study in the literature that claims the characterization of polysaccharides extracted from SCG as exposed here. Therefore, the purpose of the present study was to perform a chemical and structural characterization, as well as to determine the antioxidant and antimicrobial properties of the polysaccharides extracted from SCG by alkali pretreatment.

\section{Materials and methods}

\subsection{Raw material}

SCG were provided by the Portuguese coffee industry Nova Delta-Comércio e Indústria de Cafés S.A. (Campo Maior, Portugal). The material was dried in an oven at $60^{\circ} \mathrm{C}$ until $5 \%$ moisture content and stored for further extraction.

\subsection{Alkali pretreatment}

Polysaccharides extraction from SCG was carried out according to the method described by Simões et al. (2010) with some modifications. Briefly, previous to the extraction, the SCG (605 g) were defatted in a Soxhlet extraction system (Tecator, HT2, Netherlands) during $4 \mathrm{~h}$ using petroleum ether as solvent $(1: 5(\mathrm{w} / \mathrm{v}))$. The fat free SCG were dried at $60^{\circ} \mathrm{C}$ until constant weight and stored for the further stages. The alkali pretreatment was then performed for polysaccharides extraction by using $4 \mathrm{M}$ sodium hydroxide $(4 \mathrm{~L})$ at $25^{\circ} \mathrm{C}$ overnight $(0.02 \mathrm{M}$ sodium borohydride was also added to prevent peeling reactions and alkaline oxidation of the polysaccharides). After this time, the produced alkali extract was centrifuged at $9700 \times \mathrm{g}$ for $15 \mathrm{~min}$ at $4{ }^{\circ} \mathrm{C}$, filtered through Whatman filter paper and acidified to $\mathrm{pH} 5.0$ with glacial acetic acid. Next, the filtrate was dialyzed at $4{ }^{\circ} \mathrm{C}$ with an 8000 Da membrane for 12 days, with several distillated water changes. After dialysis, the retentate into the membrane was centrifuged at the same conditions above mentioned and the supernatant was frozen and freeze-dried. Freeze-dried powder was stored at room temperature and protected from the light and humidity until further use.

\subsection{Polysaccharide yield}

Three different extraction yields of polysaccharides were determined (Y1, Y2, and Y3), which can represent important economic parameters of the process. Y1 represents the total yield of the extraction, expressed as $\mathrm{g}$ of lyophilized material per $100 \mathrm{~g} \mathrm{SCG}$; Y2 refers to the quantity of sugars extracted and was expressed as $\mathrm{g}$ of total sugars present in the lyophilized material per $100 \mathrm{~g} \mathrm{SCG}$; finally, Y3 represents the yield of the quantity of sugar extracted with respect to total sugars existent in the SCG, which is defined as $\mathrm{g}$ of total sugars in the lyophilized material per 100 of sugars from SCG.

\subsection{Analytical methodology}

\subsubsection{Chemical characterization}

Polysaccharides recovered from SCG were submitted to a dilute acid hydrolysis with sulfuric acid ( $120 \mathrm{mg} \mathrm{H}_{2} \mathrm{SO}_{4} / \mathrm{g}$ material lyophilized). The mixture was vortexed and sterilized at $120^{\circ} \mathrm{C}$ for $20 \mathrm{~min}$. Then, sugar concentrations were made by high performance liquid chromatography (HPLC) using an equipment LC-10 A (Jasco, Japan) with a Meta Carb $87 \mathrm{P}$ column at $80^{\circ} \mathrm{C}$, ultrapure water previously boiled and degassed in a ultrasonic bath as mobile phase, and a refractive index (RI) detector. The flow rate and the injection volume were adjusted to $0.4 \mathrm{~mL} / \mathrm{min}$ and $20 \mu \mathrm{L}$, respectively. Glucose, arabinose, galactose and mannose were identified and quantified from standard curves made with known concentrations of each compound and expressed as \% mol. The response of the RI detector was recorded and integrated using the Star Chromatography Workstation software (Varian).

\subsubsection{Structural characterization}

Crystalline phases of SCG polysaccharides were evaluated by X-ray diffraction (XRD) as described by Ballesteros, Teixeira, and Mussatto (2014). The chemical groups and bonding arrangement of constituents present in the polysaccharides were determined by Fourier transform infrared spectroscopy (FTIR) using a PerkinElmer 16 PC spectrometer (Boston, USA) equipped with a diamond-composite attenuated total reflectance (ATR) cell. The measurements were recorded with a wavenumber range from 4000 to $400 \mathrm{~cm}^{-1}$ and 16 scans per sample. Differential scanning calorimetry (DSC) and thermogravimetric analyses (TGA) were carried out as previously described (Ballesteros et al., 2014).

\subsubsection{Antioxidant phenolic compounds characterization}

For the analysis of total phenolic compounds and antioxidant activity of the polysaccharides extracted from SCG by alkali pretreatment, the lyophilized material was mixed with ultrapure water in a ratio of $1 \mathrm{mg} / \mathrm{mL}$, vortexed for $1 \mathrm{~min}$ and then filtered through $0.22 \mu \mathrm{m}$ filters. Additionally, two commercial antioxidant phenolic compounds (2,6-di-tert-butyl-4-methylphenol and tertbutyl-4-methoxyphenol, known as BHT and BHA, respectively) were used as standards to evaluate the antioxidant capacity of polysaccharides and Tukey's range test was considered to evaluate significant differences $(p<0.05)$ among samples.

2.4.3.1. Phenolic compounds. The total phenolic compounds obtained from SCG were determined using the Folin-Ciocalteu reagent method adapted to a 96-well microplate (Meneses, Martins, Teixeira, \& Mussatto, 2013). The total content of phenolic compounds was expressed as milligram of gallic acid equivalent per g of dry material (mg GAE/g lyophilized).

2.4.3.2. Total antioxidant activity. The total antioxidant activity (TAA) of polysaccharides extracted from SCG was estimated as described by Prieto, Pineda, and Aguilar (1999) with some modifications. Briefly, $200 \mu \mathrm{L}$ of sample was added to a glass tube containing $2 \mathrm{~mL}$ of reagent solution $(0.6 \mathrm{M}$ sulfuric acid, $28 \mathrm{mM}$ sodium phosphate and $4 \mathrm{mM}$ ammonium molybdate). The tubes were covered and maintained during $90 \mathrm{~min}$ in a water-bath at $95^{\circ} \mathrm{C}$ and then, placed to cool at room temperature. The absorbance was measured at $695 \mathrm{~nm}$ using a spectrophotometer V-560 (Jasco, Japan) against a blank of distilled water. A calibration curve was prepared with a standard solution of $\alpha$-tocopherol $(25,75,125,250,375$ and $500 \mu \mathrm{g} / \mathrm{mL}$ ). TAA expressed as milligrams of $\alpha$-tocopherol equivalent per $\mathrm{mL}$ of extract (mg TOC/mL).

2.4.3.3. Ferric reducing antioxidant power assay. The antioxidant activity of polysaccharides extracted from SCG by the ferric 
reducing antioxidant power (FRAP) assay was determined according to the methodology described by Meneses et al. (2013). The FRAP values were expressed as milligrams of ferrous equivalent per $\mathrm{mL}$ of extract $(\mathrm{mg} \mathrm{Fe}(\mathrm{II}) / \mathrm{mL}$ ).

2.4.3.4. Free radical scavenging activity (DPPH assay). The DPPH radical scavenging activity of polysaccharides extracted from SCG was determined using the methods described by Fukumoto and Mazza (2000) and Silva et al. (2004) in combination and with some modifications. For each sample, a dilution series (four different concentrations) were prepared. The reaction was carried out in a 96-well microplate containing $25 \mu \mathrm{L}$ of sample and $200 \mu \mathrm{l}$ of $150 \mu \mathrm{M}$ DPPH solution (2,2-diphenyl-1-picrylhydrazyl dissolved in $80 \%$ methanol to an absorbance value of 0.700 at $515 \mathrm{~nm}$ ). The produced solutions were vortexed and allowed to stand for $1 \mathrm{~h}$ in the dark at room temperature. Then the absorbance was measured at $515 \mathrm{~nm}$ in a spectrophotometric microplate reader (Sunrise Tecan, Grödig, Austria) using methanol as blank. The control solution consisted in using methanol instead of the sample. The radical scavenging activity was calculated by using the Eq. (1), where $A_{c}$ and $A_{s}$ are the absorbance of the control solution and the absorbance of the sample solution, respectively. A calibration curve was prepared with a standard solution of Trolox (6-hydroxy-2,5,7,8tetramethylchroman-2-carboxylic acid) diluted in methanol (40, $80,100,300,400$ and $600 \mu \mathrm{M})$. DDPH percent inhibition data were plotted as a function of antioxidant concentration to obtain DPPH inhibition concentration at $50 \%\left(\mathrm{IC}_{50}\right)$. The $\mathrm{IC}_{50}$ values were expressed as milligrams of Trolox equivalent (TE) per $\mathrm{mL}$ of extract (mg TE/mL).

$\%$ inhibition $=(1-A s / A c) * 100$

2.4.3.5. Radical cation decolorization assay. The radical cation decolorization (ABTS) assay of polysaccharides extracted from SCG was determined as described by Re et al. (1999) and Ozgen, Reese, Tulio, Scheerens, and Miller (2006) with some modifications. Each sample was diluted to four different concentrations such that the percent inhibition was between $20 \%$ and $80 \%$. Assays were conducted by combining $130 \mu \mathrm{L}$ of sample with $3 \mathrm{~mL}$ of ABTS radical cation solution. The resulting solutions were maintained during $30 \mathrm{~min}$ in darkness at room temperature, and the absorbance was then measured at $734 \mathrm{~nm}$ using a spectrophotometer V-560 (Jasco, Japan) being distilled water used as control solution instead of the sample. ABTS radical cation was prepared by mixing $7 \mathrm{mM} \mathrm{2,2'-}$ azino-bis(3-ethylbenzothiazoline-6-sulphonic acid) diammonium salt (ABTS) dissolved in water with a $2.45 \mathrm{mM}$ potassium persulfate solution. This mixture was vortexed for $2 \mathrm{~min}$, set in ultrasonic bath during $20 \mathrm{~min}$ and then, left in the dark at $4{ }^{\circ} \mathrm{C}$ between 12 and $16 \mathrm{~h}$ for achieving a stable oxidative state. After this time, ABTS radical cation solution was diluted in a $20 \mathrm{mM}$ acetate buffer ( $\mathrm{pH} 4.5$ ) solution to an absorbance of $0.70 \pm 0.01$ at $734 \mathrm{~nm}$. A calibration curve was constructed using a standard solution of Trolox diluted in ethanol $(50,100,200,250,300,400$ and $500 \mu \mathrm{M})$. The percent inhibition of ABTS radical cation was calculated using the same equation employed in the DPPH radical scavenging. The $\mathrm{IC}_{50}$ values were expressed as milligrams of Trolox equivalent (TE) per $\mathrm{mL}$ of extract ( $\mathrm{mg} \mathrm{TE} / \mathrm{mL})$.

\subsubsection{Antimicrobial activity assays}

Antimicrobial evaluation was performed against five food pathogenic fungi that drastically influence the quality and safety of postharvest fruits (Jasso de Rodríguez et al., 2011): Alternaria sp. MUM 02.42, Cladosporium cladosporioides MUM 97.06, Phoma violacea MUM 97.08, Penicillium italicum MUM 02.25 and Penicillium expansum MUM 02.14, being obtained from the collection of the Mycology Laboratory (MUM) of the University of Minho, Portugal.
All the strains were cultured into potato dextrose agar (PDA) and incubated at $25 \pm 2{ }^{\circ} \mathrm{C}$ during 15 days before antimicrobial test.

The determination of minimal inhibitory concentration (MIC) of polysaccharides extracted from SCG was performed using the micro-dilution methodology for filamentous fungi described by the Clinical and Laboratory Standards Institute (CLSI, 2002).

\section{Results and discussion}

\subsection{Yield of extraction and chemical characterization of polysaccharides}

Table 1 shows the monosaccharide composition and extraction yield of the recovered polysaccharides. SCG are a residue rich in sugars polymerized into cellulose and hemicellulose, which correspond to $51.50 \%(\mathrm{w} / \mathrm{w})$ of its composition on a dry weight basis (Ballesteros et al., 2014). In the present study, the total sugar content extracted from SCG (lyophilized material) was 39\%, while Y1, Y2 and Y3 were 6.05, 2.38 and $4.57 \%(\mathrm{w} / \mathrm{w})$, respectively. Y1 is in agreement to the values obtained by Simões et al.(2009) when used $4 \mathrm{M} \mathrm{NaOH}$ to extract polysaccharides from SGC, in contrast toY2, which was almost 2-fold higher. Y3 was lower taking into account the high amount of polysaccharides present in the SCG (Ballesteros et al., 2014).

The chromatogram profile shown in Fig. 1a revealed glucose, galactose, arabinose and mannose as the only sugars present in SCG polysaccharide. The monosaccharide composition showed galactose $(60.27 \% \mathrm{~mol})$ as the dominant sugar, followed by arabinose $(19.93 \% \mathrm{~mol})$, glucose $(15.37 \% \mathrm{~mol})$ and mannose $(4.43 \% \mathrm{~mol})$. These results are in agreement with others studies which reported that polysaccharides in coffee wall are constituted by galactose, arabinose, mannose and glucose, forming mainly galactomannan, arabinogalactan and cellulose structures (Arya \& Rao, 2007; Ballesteros et al., 2014; Mussatto, Carneiro, et al., 2011; Simões et al., 2009). However, the obtained sugar percentages revealed differences when compared with those works, but concurred with the results reported by Simões et al. (2009), who evaluated $4 \mathrm{M}$ $\mathrm{NaOH}$ to extract polysaccharides from SGC. As a result, galactose and arabinose were the most representative sugars found in the supernatant, which is in agreement with the presented results. The quantity of mannose extracted in both cases was lower when compared with other methods used to extract polysaccharides from SCG (Ballesteros et al., 2014; Mussatto, Carneiro, et al., 2011), which indicates that a large proportion of mannose remains in SCG, suggesting that stronger conditions should be used for their extraction. For instance, mannose from SCG could be subjected to a chemical acetylation process (Simões et al., 2009, 2010) increasing thus the solubility of this sugar in water and other organic solvents, since the solvent plays an important role in the extraction process and should be chosen with respect to the organic compound of interest.

Additionally, the efficiency of the extraction depends of many factors such as solid/liquid ratio, solvent, temperature, extraction time, variety of the beans used and their degree of roasting, among others. Such parameters could be optimized, but this was not the objective of the present study.

\subsection{Structural characteristics}

\subsection{1. $X$-ray diffraction}

Fig. 1b displays the XRD patterns for SCG and the polysaccharide extracted from SCG. In order to evaluate the crystallinity of polysaccharides after alkali pretreatment, the XRD spectrum was compared with a XRD spectrum of SCG, obtained in a previous study (Ballesteros et al., 2014), in which the SCG did not suffer any chemical pretreatment before the analysis. As it can be seen, the unique 
Table 1

Monosaccharide composition and extraction yield of the polysaccharides from spent coffee grounds.

\begin{tabular}{|c|c|c|c|c|c|c|c|}
\hline \multicolumn{3}{|l|}{ Yield $^{\mathrm{a}}$} & \multicolumn{4}{|c|}{ Monosaccharide composition (\% mol) } & \multirow[t]{2}{*}{ Total sugars $(\%)$} \\
\hline Y1 & Y2 & Y3 & Arabinose & Mannose & Galactose & Glucose & \\
\hline 6.05 & 2.38 & 4.57 & $19.93 \pm 1.74$ & $4.43 \pm 0.16$ & $60.27 \pm 0.51$ & $15.37 \pm 0.93$ & $39.00 \pm 0.19$ \\
\hline
\end{tabular}

Results of monosaccharide composition are expressed as mean \pm standard deviation; $n=3$.

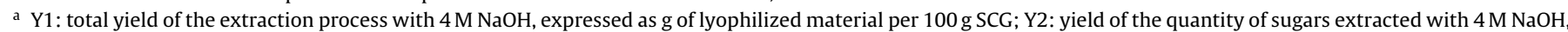

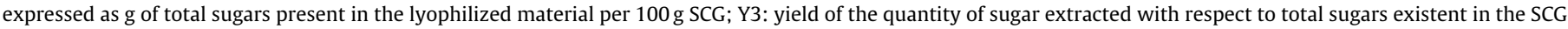
expressed as $\mathrm{g}$ of total sugars in the lyophilized material per $100 \mathrm{~g}$ of sugars from SCG.

crystalline peak in SCG corresponds to the cellulose (Ballesteros et al., 2014), while the polysaccharides extracted from SCG did not present any crystalline region. Although the chemical composition (Table 1 ) revealed glucose (cellulose) into the sugars present in the lyophilized material, the XRD spectra of polysaccharides suggests a glucose with amorphous structure, since it is known to have crystalline and amorphous regions, in contrast with hemicellulose that present an amorphous structure (Ragauskas \& Huang, 2013). This result could be related to the fact that alkali pretreatment is more suitable to extract the hemicellulose structure, being more easily degradable and susceptible to chemical attacks than cellulose (Ragauskas \& Huang, 2013).

\subsubsection{Fourier transform infrared spectroscopy}

Fig. 1c shows the FTIR analysis performed to polysaccharides extracted from SGC. The obtained spectrum corresponds to a typical carbohydrate pattern when compared with others' IR spectra of polysaccharides reported in the literature (Cerqueira et al., 2011; Ren et al., 2014; Zeng, Zhang, Gao, Jia, \& Chen, 2012). The broad peak between 3600 and $3200 \mathrm{~cm}^{-1}$ was related to the hydroxyl group of $\mathrm{O}-\mathrm{H}$ stretching vibration and the weak band between 3000 and $2800 \mathrm{~cm}^{-1}$ was attributed to $\mathrm{C}-\mathrm{H}$ stretching vibration. The region between 1700 and $1500 \mathrm{~cm}^{-1}$ was related with carbonyl groups $(\mathrm{C}=\mathrm{O})$ asymmetrical and symmetric stretching vibrations (Ren et al., 2014). This band was also highly associated with chlorogenic acids and caffeine (Ribeiro, Salva, \& Ferreira, 2010). Therefore, the peak at $1650 \mathrm{~cm}^{-1}$ could be attributed to a small absorption of these compounds, remaining from the SCG. The peak at $1374 \mathrm{~cm}^{-1}$ corresponds to $\mathrm{C}-\mathrm{H}$ in plane bending vibration (Ren et al., 2014). The sharp band between 1194 and $925 \mathrm{~cm}^{-1}$ corresponds to stretching vibration of $\mathrm{C}-\mathrm{O}$ in $\mathrm{C}-\mathrm{O}-\mathrm{H}$ bonds such as glycosidic bonds, and was related to polysaccharide sugars (Figueiró, Góes, Moreira, \& Sombra, 2004). The peaks at 1155 and $1080 \mathrm{~cm}^{-1}$ resulted from the bending vibrational modes of $\mathrm{C}-\mathrm{O}$ existing in the pyranose form (Figueiró et al., 2004), while the shoulder at $1024 \mathrm{~cm}^{-1}$ was indicated as C $-\mathrm{O}$ stretching (Ren et al., 2014). The peaks at 885 and $790 \mathrm{~cm}^{-1}$ were related to the presence of $\beta$-linked $D$-mannopyranose units and $\alpha$-linked D-galactopyranose units, respectively. These glycosidic configurations were reported in most seed galactomannans (Cerqueira et al., 2011; Figueiró et al., 2004).

\subsubsection{Thermal properties}

DSC and TGA curves (Fig. 2) were performed in order to understand the thermal behavior and chemical changes of the polysaccharides extracted from SCG. DSC thermogram exhibited two events: an initial endothermic phase followed by an exothermic phase. Thus, an early endothermic event was detected with a peak at $80.43^{\circ} \mathrm{C}$ with an associated enthalpy change of $167.30 \mathrm{~J} / \mathrm{g}$.

\section{a)}

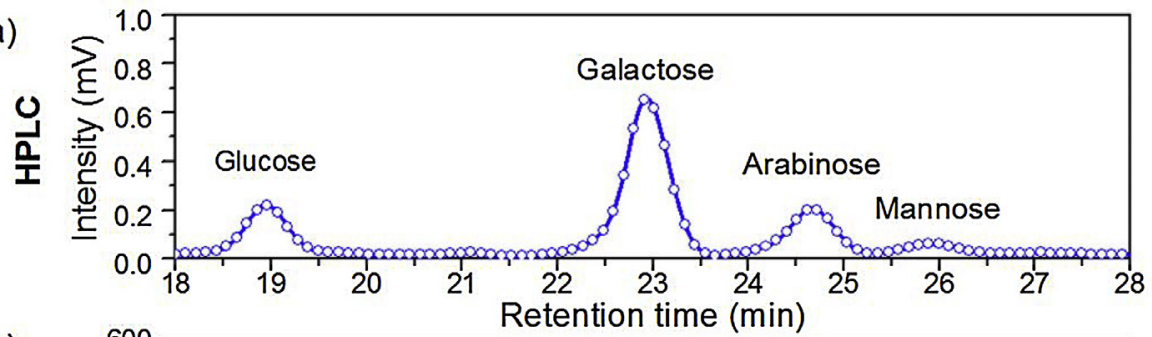

b)

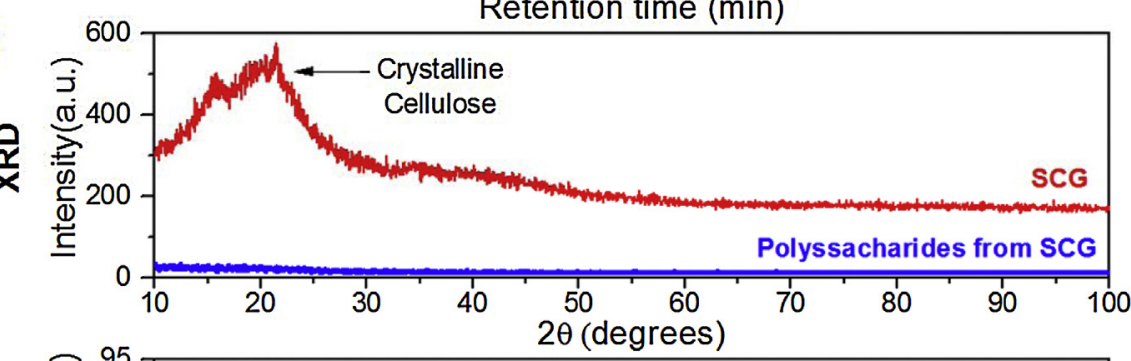

c)

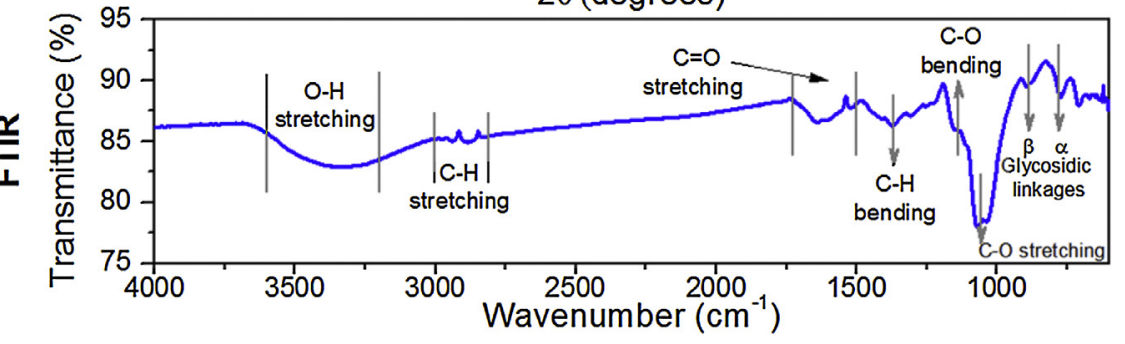

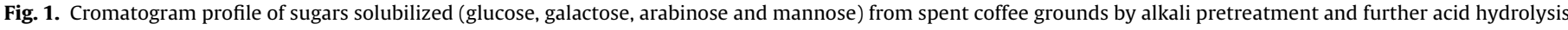

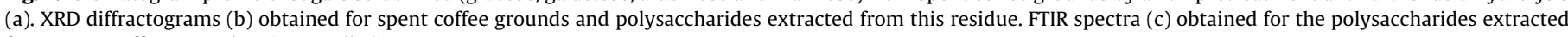
from spent coffee grounds using an alkali pretreatment. 


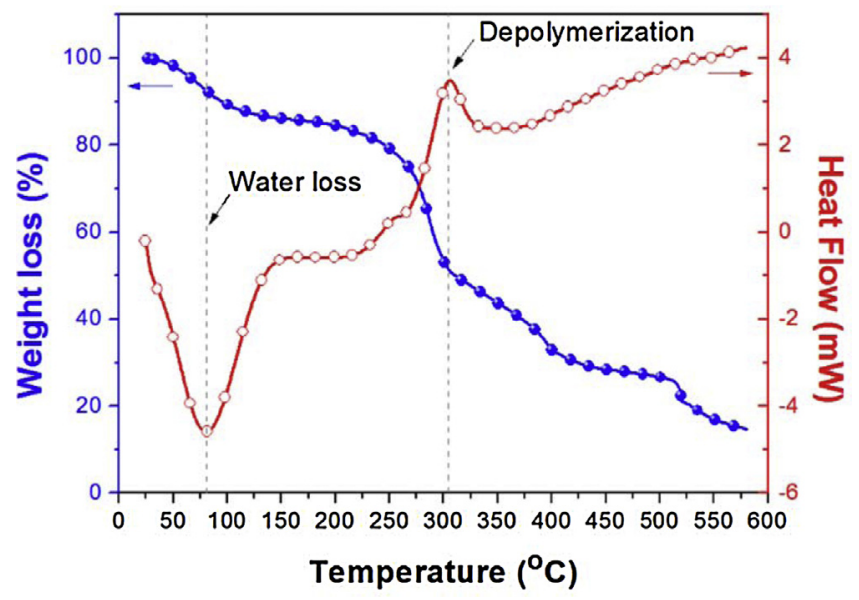

Fig. 2. TGA and DSC curves showing the thermal behavior, chemical changes and weight loss of the polysaccharides extracted from spent coffee grounds by alkali pretreatment.

This event was related to the presence of impurities in the sample and the vaporization of water (indicating the presence of hydrophilic groups), which occurs over a range of temperature. Enthalpy change in the first thermal transition was inferior when compared to those obtained for others polysaccharides (Cerqueira et al., 2011), associated to the low content of mannose:galactose ratio (Cerqueira et al., 2011; Chaires-Martínez, Salazar-Montoya, \& Ramos-Ramírez, 2008), as reported in Table 1. The second event corresponds to an exothermic transition and was observed at $303.60^{\circ} \mathrm{C}$, accompanied with an enthalpy change of $39.96 \mathrm{~J} / \mathrm{g}$. This transition was related to the thermal depolymerisation and branching of the polysaccharides, occurring at temperature ranges varying between 220 and $310^{\circ} \mathrm{C}$ (Sperling, 2006).

The TGA curve (Fig. 2) shows the weight losses of the polysaccharides when exposed to heating until $580^{\circ} \mathrm{C}$, with four defined mass loss stages. The first one started at approximately $80^{\circ} \mathrm{C}$ and corresponded to weight losses of about $12.91 \%$, resulting from the adsorbed and structural water evaporation (dehydration of the
Table 2

Total phenolic compounds and antioxidant capacity of the polysaccharides extracted from spent coffee grounds by alkali treatment.

\begin{tabular}{lr}
\hline Assay method & \multicolumn{1}{c}{ Response } \\
\hline Total phenolic compounds $(\mathrm{mg} \mathrm{GAE} / \mathrm{g}$ lyophilized) & $230.14 \pm 1.43$ \\
Total antioxidant activity $(\mathrm{mg} \mathrm{TOC} / \mathrm{mL})$ & $0.19 \pm 0.01$ \\
FRAP $(\mathrm{mg} \mathrm{Fe}(\mathrm{II}) / \mathrm{mL})$ & $0.20 \pm 0.11$ \\
DPPH IC $50(\mathrm{mg} \mathrm{TE} / \mathrm{mL})$ & $0.11 \pm 0.00$ \\
ABTS IC $50(\mathrm{mg} \mathrm{TE} / \mathrm{mL})$ & $0.08 \pm 0.00$
\end{tabular}

Results are expressed as mean \pm standard deviation; $n=3$. FRAP: antioxidant activity by the ferric reducing antioxidant power assay; DPPH: antioxidant activity by the 2,2-diphenyl-1-picrylhydrazyl assay; ABTS: antioxidant activity by 2,2'-azino-bis(3ethylbenzothiazoline-6-sulphonic acid) diammonium salt.

sample). The greatest transformation and mass losses occurred during the second stage, at approximately $300^{\circ} \mathrm{C}$. At this stage, the depolymerization and decomposition of polysaccharides occurred, providing weight losses of $37.61 \%$, in agreement with the DSC thermogram. Finally, the third and fourth thermal stages started at approximately $400^{\circ} \mathrm{C}$ and $520^{\circ} \mathrm{C}$, respectively, being related with the decomposition of the material and resulting in weight losses of $13.95 \%$ and $9.73 \%$.

\subsection{Antioxidant phenolic compounds}

In order to evaluate the phenolic compounds and the antioxidant activity of the polysaccharides extracted from SCG, aqueous extracts were obtained by mixing the lyophilized material with ultrapure water to obtain a concentration of $1 \mathrm{mg} / \mathrm{mL}$. The values obtained for the total phenolic compounds and the antioxidant activity determined by different methods are presented in Table 2. The content of phenolic compounds was higher $(230 \mathrm{mg} / \mathrm{g}$ lyophilized) when compared with other studies, which employed a conventional solid-liquid extraction method using organic solvents such as ethanol (Zuorro \& Lavecchia, 2012) and methanol (Mussatto, Ballesteros, et al., 2011) obtaining 16 mg GAE/g SCG and $21.56 \mathrm{mg}$ GAE/g SCG, respectively. The high value obtained in the present study may be related with the additional stages carried out before and after the extraction process, such as the SCG defatting and the extract lyophilization.

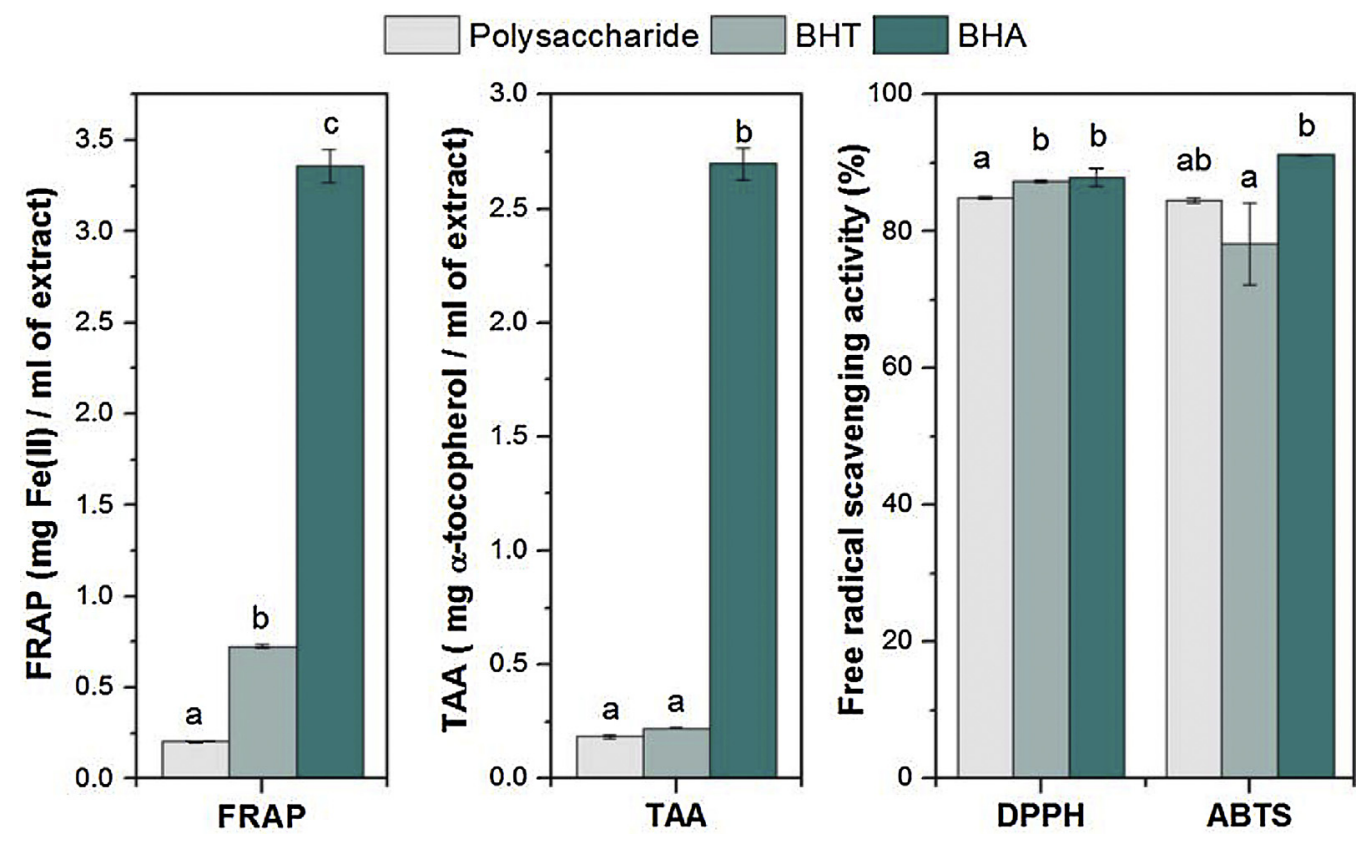

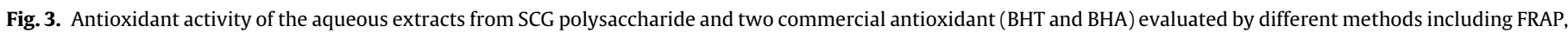
TAA, DPPH and ABTS assays. Different letters within each method mean values statistically different at 95\% confidence level. 
(a)

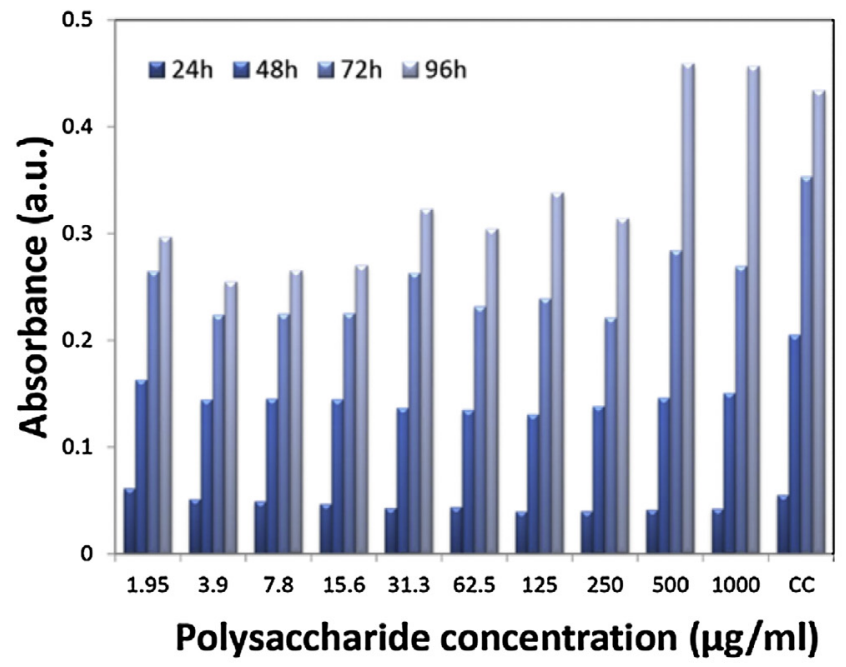

(c)

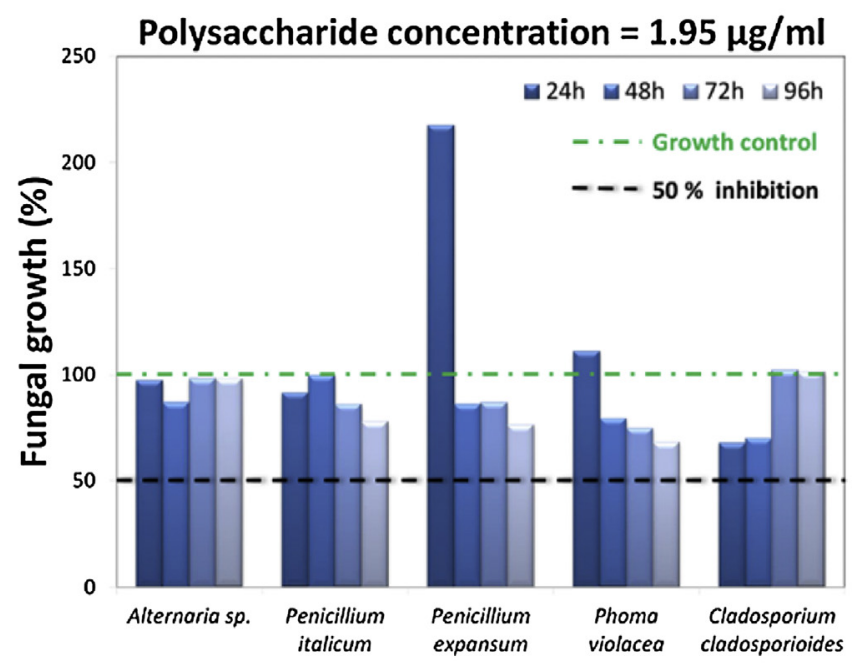

(b)

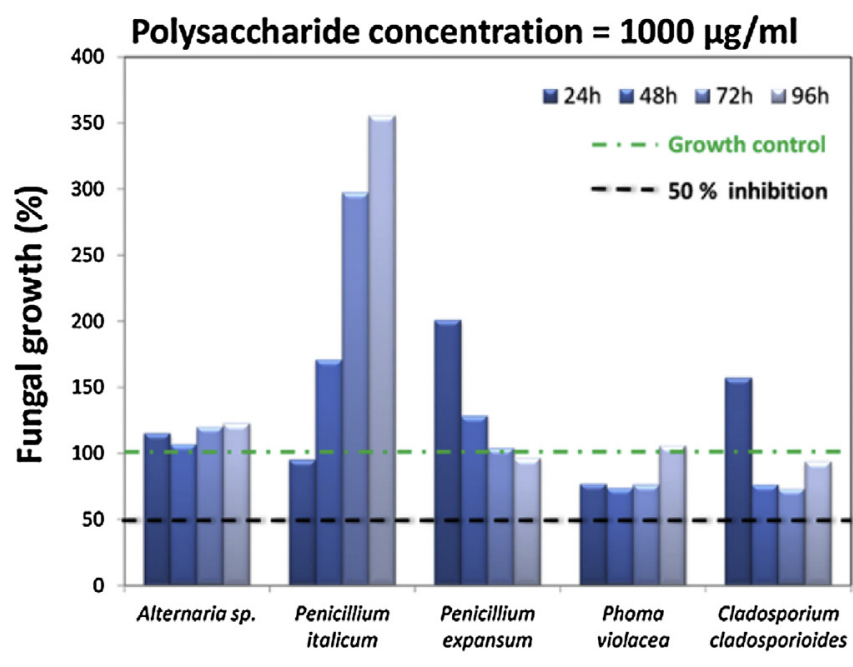

(d)

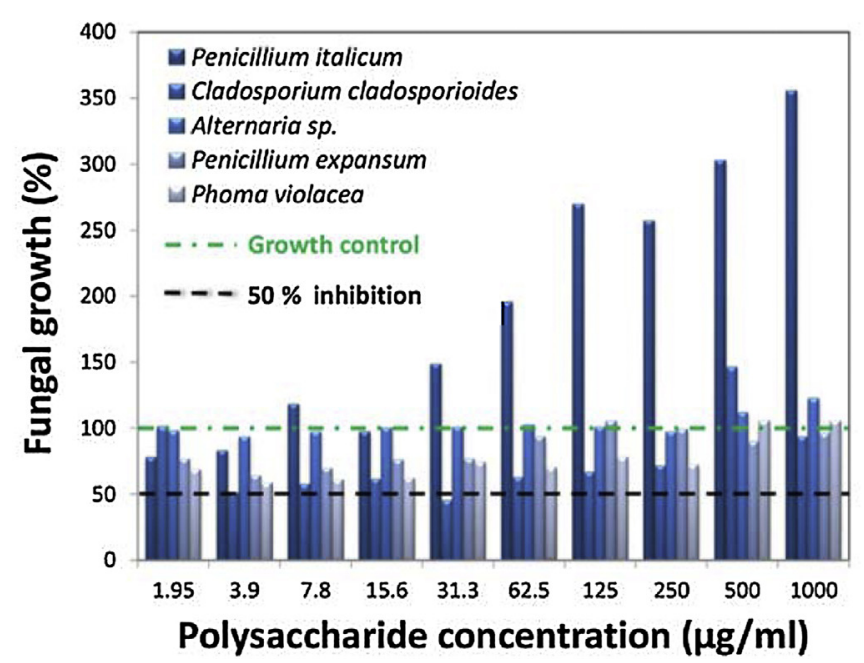

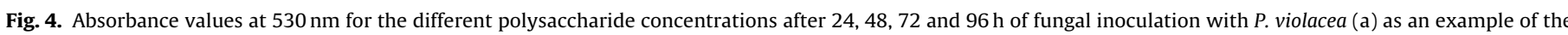

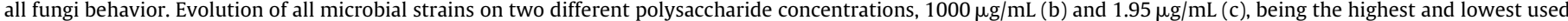

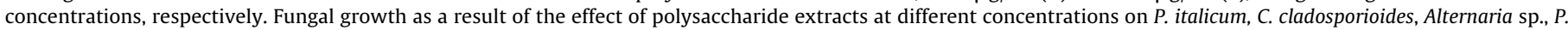

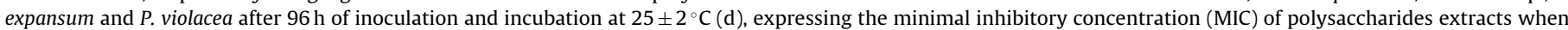
compared with a growth control.

According to the current literature, different methods can be used to evaluate the antioxidant activity in food and biological systems. However, as each method is based on a different reaction, it is strongly advisable determining the antioxidant potential of a sample by different methods in order to better interpret the results. Fig. 3 shows the antioxidant properties of polysaccharides extracted from SCG, using three different methods. The obtained values were compared with standard antioxidant compounds such as BHT and BHA, which were analyzed under the same procedure and concentration than the extracted polysaccharides. Significant differences $(p<0.05)$ were obtained when comparing the values of extracted polysaccharides and the standards. When analyzed by FRAP assay the values of BHT were 3.5-fold higher than the values obtained for extracted polysaccharides. BHA was 15-fold higher in both FRAP and TAA assays, but the polysaccharides showed a similarly antioxidant potential for TAA when compared with BHT, which was 1.2-fold higher. On the other hand, the percentage of inhibition for all samples (at concentration of
$1 \mathrm{mg} / \mathrm{mL}$ ) when analyzed by DPPH and ABTS methods was much closer to the standards, clearly seen in Fig. 3. However, it is known that the scavenging activity of compounds is directly related with the concentration, and hence for the polysaccharide concentration showing the $\mathrm{IC}_{50}$ (at concentrations of 0.7 and $0.9 \mathrm{mg} / \mathrm{mL}$, for DPPH and ABTS, respectively), BHA and BHT exhibited higher than $50 \%$ of inhibition, revealing the stronger antioxidant capabilities of the standards. Although in almost all antioxidant assays the standards showed to have higher values than polysaccharides extracted form SCG, the antioxidant activity obtained by DPPH assay revealed higher free radical scavenging activity compared with other works; e.g. polysaccharides extracted from edible mushrooms species, such as Pleurotus australis, Ileodictyon cibarium, Hericium erinaceum and Hericium coralloides need higher concentration of polysaccharide extract to achieve inhibitions at $50 \%$, being 4.03, 5.78, 5.82 and $7.19 \mathrm{mg} / \mathrm{mL}$, respectively (Ren et al., 2014). There are several factors that can influence the antioxidant activity of the extracts. For instance, defatting process, which is normally 
used to remove fatty compounds in coffee before polysaccharides extraction (Bravo, Monente, Juániz, De Peña, \& Cid, 2013; Nunes et al., 2005), may influence the antioxidant capacity of the samples since antioxidant compounds could be also removed (Bravo et al., 2013).

\subsection{Antimicrobial activity}

Polysaccharides extracted from SCG were screened for antimicrobial activity against five fungi using the micro-dilution methodology. All strains were evaluated as a function of the incubation time, assessing the growth rate after 24, 48, 72 and $96 \mathrm{~h}$ of incubation at $25 \pm 2{ }^{\circ} \mathrm{C}$. Fig. 4 a shows the absorbance values at $530 \mathrm{~nm}$ obtained for $P$. violacea as an example of the absorbance values at $530 \mathrm{~nm}$ for different polysaccharide concentrations. The graph clearly depicts a normal development of $P$. violacea growth control (CC), while clear alterations to the fungi growth are noticed when different concentrations of the polysaccharides are tested. These changes were observed in all fungi tested and were discussed in more detail using the percentage of growth, plotted in Fig. 4b and c. The evolution of all microbial strains growth on two different concentrations, $1000 \mu \mathrm{g} / \mathrm{mL}$ (maxima condition) and $1.95 \mu \mathrm{g} / \mathrm{mL}$ (minimal condition) are displayed in Fig. 4b and c, respectively. Both polysaccharide concentrations properly represented two distinctive behaviors for low and high concentrations against all fungi. Thus, when the polysaccharide concentration was lower than $31.3 \mu \mathrm{g} / \mathrm{mL}$, the trends of fungi growth with respect to time were similar and were presented in Fig. 4c, while for higher concentration the growth tendencies were presented in Fig. 4c.

Alternaria sp. exhibited very low inhibition growth for all the tested concentrations, reaching values no larger than $20 \%$ of inhibition. On the other hand, P. italicum, showed a particular behavior when compared to the growth of the control (CC), where higher polysaccharide concentrations promoted the fungal growth instead of inhibiting, attaining a growth almost 4-fold higher after $96 \mathrm{~h}$ of incubation. This behavior suggests that higher polysaccharide concentrations may act as a carbon source, stimulating the $P$. italicum growth in greater proportions than the culture media. On the contrary, for lower concentrations, the fungus growth was slightly inhibited up to value no greater than $25 \%$. P. expansum exhibited higher growth rates for the first $24 \mathrm{~h}$ compared to the growth control. However, a monotonic reduction of fungus growth was observed when the experiment time increased for all the concentrations, achieving higher inhibition values for lower polysaccharide concentrations. The extracted polysaccharide presented the higher inhibition efficacy for $P$. violacea and $C$. cladosporioides among all the strains tested. At higher polysaccharides doses, the $P$. violacea showed a constant inhibition, reducing to $0 \%$ of inhibition after $96 \mathrm{~h}$ of incubation, indicating a short-term inhibition that may be due to the consumption of the inhibitory components present in the extracted polysaccharide. Nonetheless, for lower doses, an increase of the growth inhibition was observed. This behavior was also observed for $C$. cladosporioides at some concentrations, but with less defined tendencies in this particular strain. The differences between the high and low polysaccharide concentrations suggest a competition between the antimicrobial components in the extracts and the increased carbon source that the polysaccharide may offer to the microorganism, limiting the extracts function as an antimicrobial agent to low polysaccharide concentrations.

The previous behavior could be more clearly observed in Fig. $4 \mathrm{~d}$, where the growth percentage for all the strains at $96 \mathrm{~h}$ is plotted as a function of the polysaccharide concentration. This figure evidences that the increment of the polysaccharide doses reduced the inhibition of growth for the five different fungi strains. As previously mentioned, Alternaria sp. did not show significant
Table 3

Optimal conditions and percent inhibition of the polysaccharide extracts on growth of different microbial strains.

\begin{tabular}{lcc}
\hline Microbial strains & Optimal conditions $^{\mathrm{a}}(\mu \mathrm{g} / \mathrm{mL})$ & Percent inhibition $(\%)$ \\
\hline Penicillium italicum & 1.95 & $22.04 \pm 4.98$ \\
& 3.9 & $17.03 \pm 4.89$ \\
Cladosporium & 31.3 & $54.60 \pm 7.06$ \\
cladosporioides & 3.9 & $48.63 \pm 9.84$ \\
Alternaria sp. & 3.9 & $6.62 \pm 0.73$ \\
& 7.8 & $2.78 \pm 0.18$ \\
Penicillium expansum & 3.9 & $36.08 \pm 5.60$ \\
& 7.8 & $30.48 \pm 5.75$ \\
Phoma violacea & 3.9 & $41.27 \pm 6.95$ \\
& 7.8 & $38.89 \pm 4.49$ \\
\hline
\end{tabular}

a Results of the two better concentrations for each fungus. Percent inhibition was expressed as mean \pm standard deviation; $n=6$.

changes among the concentrations studied, indicating the lack of interaction between the extract and the strain. $C$. cladosporioides exhibited the highest inhibition at $31.3 \mu \mathrm{g} / \mathrm{mL}$ (54.60\%), as shown in Table 3. Concentrations of $3.9 \mu \mathrm{g} / \mathrm{mL}$ showed high percent of inhibition, being the concentration in which the five strains revealed higher antimicrobial activity. The evolution of the growth inhibition as a function of time is exposed in Fig. 5, confirming the facts previously described. Whereas the optimal conditions and percent inhibition of the polysaccharide extracts after $96 \mathrm{~h}$ of incubation are shown in Table 3 for the different microbial strains.

Additionally, tests with a known antimicrobial agent (fluconazole) revealed antimicrobial behavior (50\% of growth inhibition or more) for concentrations larger than $50 \mu \mathrm{g} / \mathrm{mL}$ for Alternaria sp and C. cladosporioides, and $100 \mu \mathrm{g} / \mathrm{mL}$ for $P$. italicum and $P$. violacea, without evidence of the antimicrobial effect on the P. expansusm, where the growth inhibition was no higher than $30 \%$.

Although the antimicrobial effect of extract rich in polysaccharides is not well understood, some authors have proposed that the polysaccharide may act as an external barrier, blocking the essential nutrients, impeding the microbial growth (Ren et al., 2014). Nevertheless, this barrier behavior should be increased as the polysaccharide concentration is increased, contradicting the results found in this report, where for higher concentration the antimicrobial effect is not evidenced. As a result, probably the antimicrobial effect of the extract may be due to residual components such as phenolic compounds (Jasso de Rodríguez et al.,

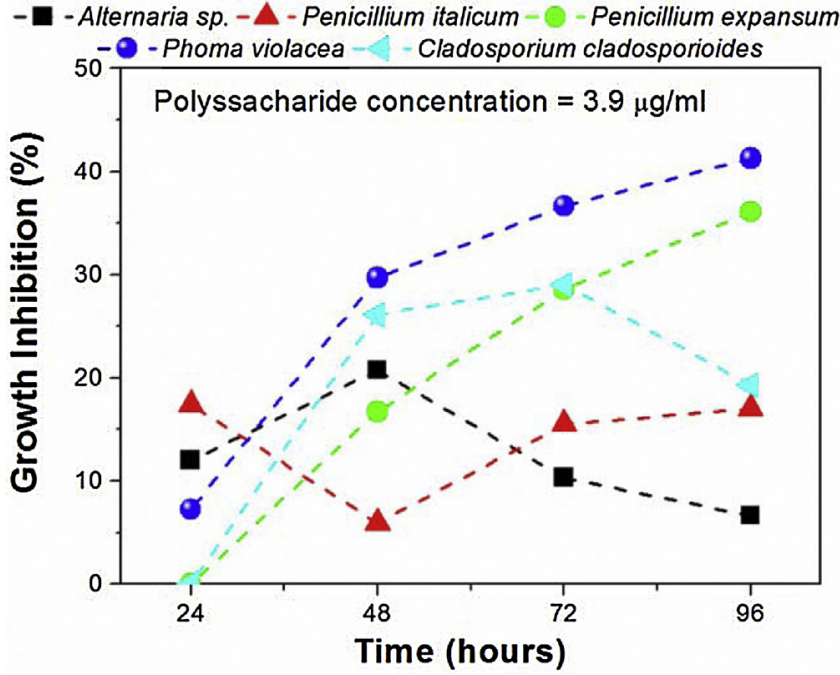

Fig. 5. Growth inhibition percentage of the polysaccharide concentration at $3.9 \mu \mathrm{g} / \mathrm{mL}$, as a function of time, revealing higher antimicrobial activity against the five tested stains (Alternaria sp., P. italicum, P. expansum, Phoma violacea and Cladosporium cladosporioides). 
2011) that are retained in the extract during the process, which compete between the polysaccharide as a carbon source for high concentrations.

\section{Conclusions}

The alkali pretreatment using $4 \mathrm{M} \mathrm{NaOH}$ as solvent showed to be a good option for an efficient extraction of polysaccharides from SGC. The most relevant sugars in SCG polysaccharides were galactose, followed by arabinose, glucose and mannose. Polysaccharides were thermostable in a large range of temperature, being therefore suitable for application in the manufacture of biomaterials and encapsulation products for several industrial purposes. Additionally, they revealed good antioxidant activity through different methods and presented high antimicrobial percent inhibition against $P$. violacea and $C$. cladosporioides. These findings open up possibilities to evaluate SGC polysaccharides as bioactive compounds in different food and pharmaceutical applications.

\section{Acknowledgements}

The authors acknowledge the financial support of the Science and Technology Foundation of Portugal (grant SFRH/BD/80948/2011) and the Strategic Project PEstOE/EQB/LA0023/2013. The authors also thank the Project "BioInd - Biotechnology and Bioengineering for improved Industrial and Agro-Food processes", REF. NORTE-07-0124-FEDER-000028 Co-funded by the Programa Operacional Regional do Norte (ON.2 - O Novo Norte), QREN, FEDER.

\section{References}

Arya, M., \& Rao, L. J. M. (2007). An impression of coffee carbohydrates. Critical Reviews in Food Science and Nutrition, 47(1), 51-67.

Ballesteros, L. F., Teixeira, J. A., \& Mussatto, S. I. (2014). Chemical, functional, and structural properties of spent coffee grounds and coffee silverskin. Food and Bioprocess Technology, 7, 3493-3503.

Bravo, J., Monente, C., Juániz, I., Paz De Peña, M., \& Cid, C. (2013). Influence of extraction process on antioxidant capacity of spent coffee. Food Research International, 50(2), 610-616.

Cerqueira, M. A., Pinheiro, A. C., Souza, B. W. S., Lima, Á. M. P., Ribeiro, C., Miranda, C., et al. (2009). Extraction, purification and characterization of galactomannans from non-traditional sources. Carbohydrate Polymers, 75(3), 408-414.

Cerqueira, M. A., Souza, B. W. S., Simões, J., Teixeira, J. A., Domingues, M. R. M., Coimbra, M. A., et al. (2011). Structural and thermal characterization of galactomannans from non-conventional sources. Carbohydrate Polymers, 83(1), $179-185$.

Chaires-Martínez, L., Salazar-Montoya, J. A., \& Ramos-Ramírez, E. G. (2008). Physicochemical and functional characterization of the galactomannan obtained from mesquite seeds (Prosopis pallida). European Food Research and Technology, 227(6), 1669-1676.

CLSI - Clinical and Laboratory Standards Institute. (2002). Reference method for broth dilution antifungal susceptibility testing of filamentous fungi. Approved standard. Document M38-A. Wayne, PA: CLSI.

Figueiró, S. D., Góes, J. C., Moreira, R. A., \& Sombra, A. S. B. (2004). On the physic-chemical and dieletric properties of glutaraldehyde crosslinked galactomannan-collagen films. Carbohydrate Polymers, 56(3), 313-320.

Fischer, M., Reimann, S., Trovato, V., \& Redgwell, R. J. (2001). Polysaccharides of green Arabica and Robusta coffee beans. Carbohydrate Research, 330(1), 93-101.

Fukumoto, L. R., \& Mazza, G. (2000). Assessing antioxidant and prooxidant activities of phenolic compounds. Journal of Agricultural and Food Chemistry, 48(8), 3597-3604

Gniechwitz, D., Reichardt, N., Blaut, M., Steinhart, H., \& Bunzel, M. (2007). Dietary fiber from coffee beverage: Degradation by human fecal microbiota. Journal of Agricultural and Food Chemistry, 55(17), 6989-6996.

Jasso de Rodríguez, D., Rodriguez García, R., Hernandez Castillo, F. D., Aguilar González, C. N., Galindo, A. S., Villarreal Quintanilla, J. A., et al. (2011). In vitro antifungal activity of extracts of Mexican Chihuahuan desert plants against postharvest fruit fungi. Industrial Crops and Products, 34(1), 960-966.

Machado, E. M. S., Rodriguez-Jasso, R. M., Teixeira, J. A., \& Mussatto, S. I. (2012). Growth of fungal strains on coffee industry residues with removal of polyphenolic compounds. Biochemical Engineering Journal, 60, 87-90.

Meneses, N. G. T., Martins, S., Teixeira, J. A., \& Mussatto, S. I. (2013). Influence of extraction solvents on the recovery of antioxidant phenolic compounds from brewer's spent grains. Separation and Purification Technology, 108, 152-158.

Mussatto, S. I. (2015). Generating biomedical polyphenolic compounds from spent coffee or silverskin. In V. R. Preedy (Ed.), Coffee in health and disease prevention (pp. 93-106). Elsevier.

Mussatto, S. I., Ballesteros, L. F., Martins, S., \& Teixeira, J. A. (2011). Extraction of antioxidant phenolic compounds from spent coffee grounds. Separation and Purification Technology, 83, 173-179.

Mussatto, S. I., Carneiro, L. M., Silva, J. P. A., Roberto, I. C., \& Teixeira, J. A. (2011). A study on chemical constituents and sugars extraction from spent coffee grounds. Carbohydrate Polymers, 83(2), 368-374.

Mussatto, S. I., Machado, E. M. S., Carneiro, L. M., \& Teixeira, J. A. (2012). Sugars metabolism and ethanol production by different yeast strains from coffee industry wastes hydrolysates. Applied Energy, 92, 763-768.

Mussatto, S. I., Machado, E. M. S., Martins, S., \& Teixeira, J. A. (2011). Production, composition, and application of coffee and its industrial residues. Food and Bioprocess Technology, 4(5), 661-672.

Nunes, F. M., Domingues, M. R., \& Coimbra, M. A. (2005). Arabinosyl and glucosy residues as structural features of acetylated galactomannans from green and roasted coffee infusions. Carbohydrate Research, 340(10), 1689-1698.

Ozgen, M., Reese, R. N., Tulio, A. Z., Jr., Scheerens, J. C., \& Miller, A. R. (2006). Modified 2 2-azino-bis-3-ethylbenzothiazoline-6-sulfonic acid (ABTS) method to measure antioxidant capacity of selected small fruits and comparison to ferric reducing antioxidant power (FRAP) and 2, 2'-diphenyl-1-picrylhydrazyl (DPPH) methods. Journal of Agricultural and Food Chemistry, 54(4), 1151-1157.

Passos, C. P., \& Coimbra, M. A. (2013). Microwave superheated water extraction of polysaccharides from spent coffee grounds. Carbohydrate Polymers, 94(1), 626-633.

Prieto, P., Pineda, M., \& Aguilar, M. (1999). Spectrophotometric quantitation of antioxidant capacity through the formation of a phosphomolybdenum complex: Specific application to the determination of vitamin E. Analytical Biochemistry 269(2), 337-341.

Ragauskas, A. J., \& Huang, F. (2013). Chemical pretreatment techniques for biofuels and biorefineries from softwood. Berlin: Springer-Verlag.

Re, R., Pellegrini, N., Proteggente, A., Pannala, A., Yang, M., \& Rice-Evans, C. (1999). Antioxidant activity applying an improved ABTS radical cation decolorization assay. Free Radical Biology and Medicine, 26(9), 1231-1237.

Ren, L., Hemar, Y., Perera, C. O., Lewis, G., Krissansen, G. W., \& Buchanan, P. K. (2014) Antibacterial and antioxidant activities of aqueous extracts of eight edible mushrooms. Bioactive Carbohydrates and Dietary Fibre, 3(2), 41-51.

Ribeiro, J. S., Salva, T. J., \& Ferreira, M. M. C. (2010). Chemometric studies for quality control of processed Brazilian coffees using drifts. Journal of Food Quality, 33(2), 212-227.

Sampaio, A., Dragone, G., Vilanova, M., Oliveira, J. M., Teixeira, J. A., \& Mussatto, S. I. (2013). Production, chemical characterization, and sensory profile of a novel spirit elaborated from spent coffee ground. LWT - Food Science and Technology, 54(2), 557-563.

Silva, B. M., Andrade, P. B., Valentão, P., Ferreres, F., Seabra, R. M., \& Ferreira, M. A. (2004). Quince (Cydonia oblonga Miller) fruit (pulp, peel, and seed) and jam: Antioxidant activity. Journal of Agricultural and Food Chemistry, 52(15), 4705-4712.

Silva, M. A., Nebra, S. A., Machado Silva, M. J., \& Sanchez, C. G. (1998). The use of biomass residues in the Brazilian soluble coffee industry. Biomass and Bioenergy 14(5-6), 457-467.

Simões, J., Madureira, P., Nunes, F. M., Rosário Domingues, M., Vilanova, M., \& Coimbra, M. A. (2009). Immunostimulatory properties of coffee mannans. Molecular Nutrition \&' Food Research, 53(8), 1036-1043.

Simões, J., Nunes, F. M., Domingues, M. R. M., \& Coimbra, M. A. (2010). Structura features of partially acetylated coffee galactomannans presenting immunostimulatory activity. Carbohydrate Polymers, 79(2), 397-402.

Sperling, L. H. (2006). Introduction to physical polymer science. New Jersey: John Wiley \& Sons, Inc

Vieira, Í. G. P., Mendes, F. N. P., Gallão, M. I., \& Brito, E. S. (2007). NMR study of galactomannans from the seeds of mesquite tree (Prosopis juliflora (Sw) DC). Food Chemistry, 101(1), 70-73.

Zeng, W.-C., Zhang, Z., Gao, H., Jia, L.-R., \& Chen, W.-Y. (2012). Characterization of antioxidant polysaccharides from Auricularia auricular using microwaveassisted extraction. Carbohydrate Polymers, 89(2), 694-700.

Zuorro, A., \& Lavecchia, R. (2012). Spent coffee grounds as a valuable source of phenolic compounds and bioenergy. Journal of Cleaner Production, 34, 49-56. 